Radiologe 2021 · 61:300-306

https://doi.org/10.1007/s00117-020-00793-0

Angenommen: 11. Dezember 2020

Online publiziert: 8. Januar 2021

(c) Springer Medizin Verlag GmbH, ein Teil von Springer Nature 2021

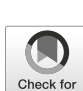

Alexey Surov - Christine March - Maciej Pech

Klinik für Radiologie und Nuklearmedizin, Otto-von-Guericke Universität Magdeburg, Magdeburg, Deutschland

\title{
Curriculare Lehre während der COVID-19-Pandemie
}

\section{Evaluation eines onlinebasierten Lehrkonzepts}

rierung der universitären Lehre im Fach Radiologie im Rahmen des Kurses „Bildgebende Verfahren“ in der COVID-19Pandemie zu dokumentieren und die Ergebnisse einer Befragung unter Studierenden im Fach Radiologie zu präsentieren.

\section{Studiendesign und Untersuchungsmethoden}

\section{Kursgestaltung}

Der verpflichtende Kurs „Bildgebende Verfahren“ der Medizinischen Fakultät des Universitätsklinikums Magdeburg findet jedes Sommersemester im 3. Studienjahr (1. klinisches Studienjahr) statt und umfasst 12 Seminare mit den Themen Mammographie, Ultraschall, Angiographie und Intervention, Thorax, Skelett, Abdomen, Neuroradiologie, Nuklearmedizin, Strahlentherapie (klinischer Strahlenschutz, klinische Strahlentherapie) sowie Physik (Strahlenschutz, bildgebende Verfahren).

Im Sommersemester 2020 fand der Kurs über einen Zeitraum von 11 Wochen statt. Für die erfolgreiche Teilnahme musste die mindestens 85 \%ige Anwesenheit nachgewiesen und eine 60-minütige Multiple-choice-Klausur am Ende des Semesters bestanden werden.

In vorherigen Semestern mit regulärem Präsenzunterricht betrug die Seminardauer $90 \mathrm{~min}$, und es wurden Vorträge mittels Power-Point-Präsentationen gehalten. Aufgrund der bestehenden Kontaktbeschränkungen stellten wir das Konzept um.
Im Gegensatz dazu fanden die Seminare nun als Videokonferenz mittels zoom $^{\circledR}$ (Zoom Video Communications, San José, CA, USA) statt. Im Vorfeld sollten sich die teilnehmenden Studierenden mittels unmittelbar vor Beginn des Sommersemesters von den Seminardozenten aufgenommener kommentierter Videopräsentationen mit den Seminarinhalten vertraut machen.

\section{Erstellung der Video- präsentationen}

Die Videopräsentationen wurden explizit in Hinblick auf die neuen Lehrverordnungen zur Reduktion der Präsenzlehre während der COVID-19-Pandemie erstellt. Zusätzlich wurden drei ergänzende Lehrvideos zur Verfügung gestellt.

Dabei wurde es den Dozenten freigestellt, ob sie den Lehrinhalt in einem oder mehreren unterteilten Videos aufnehmen wollten. Die Gesamtdauer der Videos eines Seminarthemas sollte im Idealfall $60 \mathrm{~min}$ nicht wesentlich überschreiten.

Die Videos wurden auf einer Videoplattform (Mediasite ${ }^{\circledR}$, Sonic Foundry, Madison, WI, USA) hochgeladen und anschließend auf der Lehrplattform der Universität verlinkt (Moodle ${ }^{\circledR} 3.6$, free and open-source, Moodle Pty Ltd, West Perth, Australia) und konnten in der 0,5- bis 2,0-fachen Geschwindigkeit angesehen werden.

Zusätzlich wurden von einem Drittel der Dozenten Fälle und Fragen auf der Lernplattform zur Bearbeitung zur Ver- 


\section{Der Zeitaufwand für die Beschäftigung mit den Videopräsentationen ist angemessen.}

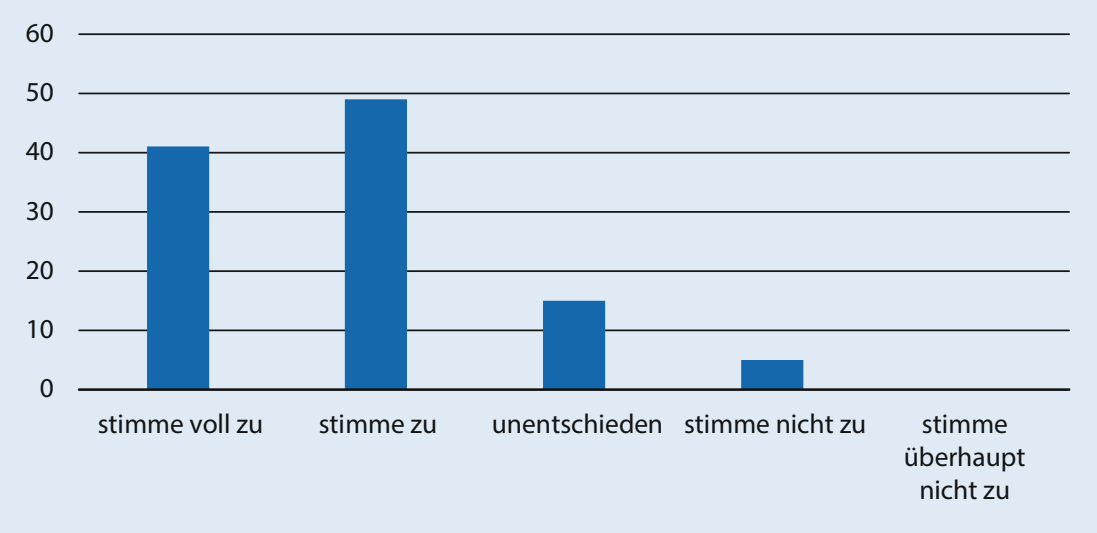

Abb. 1 ॥ Prozentuale Verteilung der Antworten auf die Aussage: „Der Zeitaufwand für die Beschäftigung mit den Präsentationen ist angemessen."

\section{Kürzere Videoabschnitte erleichtern das Lernen.}

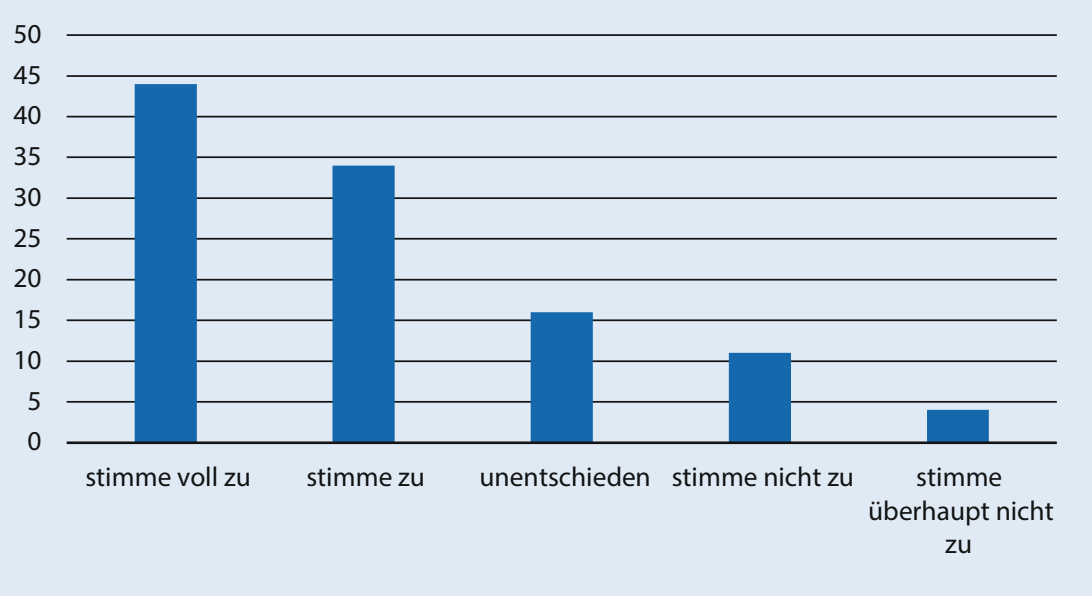

Abb. 2 ^ Prozentuale Verteilung der Antworten aufdie Aussage: „Kürzere Videoabschnitteerleichtern das Lernen."

fügung gestellt. Diese wurden anschlieBend im Onlineseminar besprochen.

\section{Organisation der Video- konferenzseminare}

Ein wöchentlicher bis zweiwöchentlicher Rhythmus der Videokonferenzseminare wurde wie in vorherigen Semestern beibehalten, allerdings wurde die Dauer des Seminars von 90 auf 45 min verkürzt, da die Videopräsentationen bereits einen Teil des Lehrinhalts vermitteln sollten.

Die Videokonferenzseminare wurden von Dozenten mit größtenteils mehrjähriger Lehrtätigkeit veranstaltet. Unter den einmal vertieft auf einzelne Videoinhalte eingegangen.

Sowohl Dozenten als auch Studierende hatten die Möglichkeit zur Monitorfreigabe. Parallel zum Seminar wurde die Chatfunktion des genutzten Videokonferenzprogramms $\left(\right.$ zoom $\left.^{\circledast}\right)$ verwendet. Die Monitorfreigabe bot die Möglichkeit, Untersuchungsbilder im Detail zu besprechen und auf Pathologien gezielt hinzuweisen. Die Darstellungen konnten beliebig vergrößert werden.

\section{Evaluation}

Fünf Wochen nach Beginn des Semesters wurde eine Onlinebefragung durchgeführt, welche die Studierenden über einen Zeitraum von 3,5 Wochen beantworten konnten. Die Studierenden konnten die Evaluation über die Lehrplattform der Medizinischen Fakultät (Moodle ${ }^{\circledR}$ ) aufrufen und wurden per E-Mail gebeten, an der Befragung teilzunehmen. Die Studierenden sollten den Kurs unter anderem im Hinblick auf den zeitlichen Aufwand, Abrufbarkeit der Videopräsentationen, Gestaltung der Videokonferenzen und generellen Aufbau des digitalen Lehrkonzepts bewerten. Insgesamt wurden 12 Fragen gestellt, die mit den folgenden Vorgaben: „stimme voll zu“, „stimme zu“, „unentschieden“, „stimme nicht zu“ oder „stimme überhaupt nicht $\mathrm{zu}^{\text {" }}$ beantwortet werden konnten. $\mathrm{Zu}$ dem war ein Feld für freie Kommentare/ Anmerkungen vorgesehen.

\section{Videoanalyse}

Mittels eines Analysetools der genutzten Onlineplattform, auf welcher die Videopräsentationen hochgeladen wurden $\left(\right.$ Mediasite $\left.^{\circledR}\right)$, konnten die Präsentationen analysiert werden. Hier konzentrierten wir uns auf die Abrufzahlen, durchschnittliche Videodauer und durchschnittliche Sehdauer der Studierenden. Die weitere Auswertung erfolgte mittels IBM SPSS Statistics for Windows, Version 26.0 (Armonk, NY, USA). Werte von $p<0,05$ wurden als statistisch signifikant angesehen. 
Radiologe 2021 · 61:300-306 https://doi.org/10.1007/s00117-020-00793-0

(c) Springer Medizin Verlag GmbH, ein Teil von Springer Nature 2021

\section{A. Surov · C. March · M. Pech}

\section{Curriculare Lehre während der COVID-19-Pandemie. Evaluation eines onlinebasierten Lehrkonzepts}

\section{Zusammenfassung}

Hintergrund. Die COVID-19-Pandemie stellt nicht nur das Gesundheitssystem und die Wirtschaft vor große Herausforderungen, sondern auch die universitäre Lehre. Ein Großteil des Unterrrichts wurde auf onlinebasierte Lehre umgestellt. Bislang ist jedoch unklar, wie diese Umstellung von den Studierenden aufgenommen wurde. Ziel dieser Arbeit war daher die Dokumentation und Evaluation eines onlinebasierten Lehrkonzepts der universitären Lehre im Fach Radiologie in der COVID-19-Pandemie. Material und Methoden. Basis des Lehrkonzepts bildete eine Kombination aus 12 Onlineseminaren des 11-wöchigen Pflichtkurses „Bildgebende Verfahren“ und 31 zugehörigen Videopräsentationen zur Vorbereitung. Fünf Wochen nach Beginn des Sommersemesters 2020 führten wir eine anonyme Onlineumfrage unter den
Teilnehmern des Kurses durch. Es wurden 12 Fragen zum Aufbau des Kurses gestellt sowie Freitextantworten in die Auswertung miteinbezogen. Zusätzlich wurden Abrufzahlen und Dauer der Videopräsentationen sowie die durchschnittliche Sehdauer analysiert. Ergebnisse. Insgesamt 110 von 213 Studierenden nahmen an der Befragung teil (51,6\%). Die Mehrheit gab an, dass das Lehrkonzept ihr Interesse an der Radiologie geweckt (72,7\%) und sie zum Selbststudium animiert hätte (78,2 \%). Zusätzlich ermöglichte es zeitlich und örtlich unabhängiges Lernen (95,5\%). Der Zeitaufwand für die Beschäftigung mit den Videopräsentationen wurde als angemessen angesehen, und kürzere Videoabschnitte würden dabei das Lernen erleichtern (70,9\%). Die optimale Dauer der Videos sollte bei ca. 10-20 min liegen. Die Mehrheit (80,9\%) wünschte sich die
Beibehaltung des Lehrkonzeptes auch für kommende Semester nach einer Aufhebung der Kontaktbeschränkungen.

Diskussion. Das onlinebasierte Lehrkonzept im Fach Radiologie während der COVID19-Pandemie genießt eine hohe Akzeptanz unter den Studierenden. Es bietet Vorteile wie ortsunabhängiges Lernen und bessere Möglichkeiten des Selbststudiums und sollte laut einer Mehrheit der befragten Studierenden in kommenden Semestern auch nach Aufhebung der Kontaktbeschränkungen beibehalten werden.

\section{Schlüsselwörter}

Kontaktbeschränkung · Onlinelehre . Lehrevaluation · Videopräsentation · Flipped classroom

\section{Curricular teaching during the COVID-19-pandemic. Evaluation of an online-based teaching concept}

\section{Abstract}

Background. The COVID-19 pandemic has created multiple challenges for health care, social and economic systems worldwide, but also for school and university teaching. Most learning has been shifted to online classes. So far, it is unclear whether these changes are well received within the student body. Objectives. The aim of this study was the evaluation of a newly developed onlinebased teaching concept for medical students studying radiology during the COVID-19 pandemic

Materials and methods. The concept consisted of 12 online seminars of the 11week course "Imaging Procedures", which were combined with 31 prerecorded video presentations. Five weeks after the start of the summer term, we conducted an anonymous online survey, which consisted of 12 questions and a box for additional comments. Furthermore, we analysed the number of requests of the presentations, the duration and the average viewing time. Results. In all, 110 of 213 students (51.6\%) completed the survey; $72.7 \%$ stated that the new teaching concept drew their interest for radiology, $78.2 \%$ agreed that it incited selfstudy as well as would facilitate time- and place-independent learning (95.5\%). The majority indicated that the time investment for the video presentations was appropriate, but shorter video sections would simplify the learning process $(70.9 \%)$ and the ideal timeframe would be $10-20 \mathrm{~min}$. The majority
(80.9\%) agreed that the concept should be maintained in upcoming semesters. Conclusions. This newly developed onlinebased teaching concept enjoys high acceptance rates among students. It offers advantages such as time- and locationindependent learning, possibilities for selfstudy and should be maintained in upcoming semesters after the end of social distancing due to the COVID-19 pandemic.

\section{Keywords}

Social distancing $\cdot$ Online-based teaching Course evaluation - Video presentation . Flipped classroom

\section{Ergebnisse}

Im Pflichtkurs waren insgesamt 213 Studierende des dritten Studienjahres der Humanmedizin eingeschrieben. Diese waren in 11 Seminargruppen à 17-22 Studierende unterteilt. Insgesamt beantworteten 110 Studierende $(51,6 \%)$ den online zur Verfügung gestellten Fragebogen.

Die Mehrheit der Teilnehmer bewertete das Abrufen der Videopräsentatio- nen als unkompliziert (87,3\% stimmten [voll] zu) und äußerte sich positiv bezüglich des vorausgesetzten Wissensniveaus der Videopräsentationen (92,7\% stimmten [voll] $\mathrm{zu}$ ).

Insgesamt wurden 28 Videopräsentationen erstellt. Für das Seminarthema Strahlentherapie wurden 3 bereits zuvor genutzte Videopräsentationen ergänzt. Für die Hälfte der Seminarthemen wurde jeweils ein Gesamtvideo aufgenommen.
Bei den übrigen Seminaren bewegte sich die Videoanzahl zwischen 2 (Skelett) bis 7 Videos (Thorax; Durchschnitt: 4,2). Die durchschnittliche Dauer der Videos betrug ca. $22 \mathrm{~min}$ ( $21 \mathrm{~min} 39 \mathrm{~s}$ ). Die durchschnittliche Gesamtvideodauer pro Seminarthema betrug ca. $51 \mathrm{~min}$ (50 min $37 \mathrm{~s}$ ). Die kürzeste Videopräsentation als Gesamtvideo dauerte ca. $17 \mathrm{~min}$ (16:42 $\mathrm{min}$ ), die längste beim Seminarthema Angiographie und In- 


\section{Was wäre die optimale Dauer eines Videoabschnitts?}

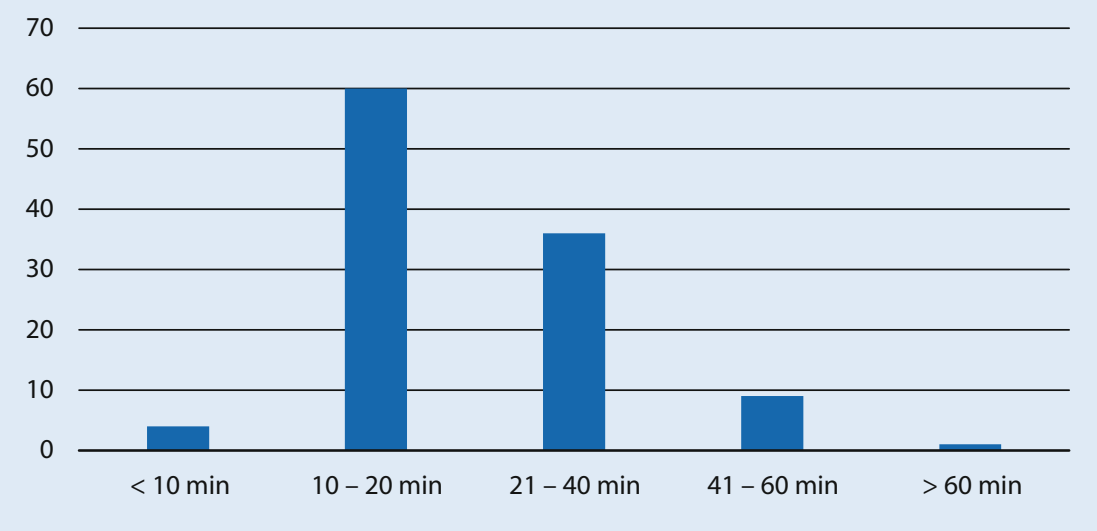

Abb. 3 ॥ Prozentuale Verteilung der Antworten auf die Frage: „Was wäre die optimale Dauer eines Videoabschnitts?"

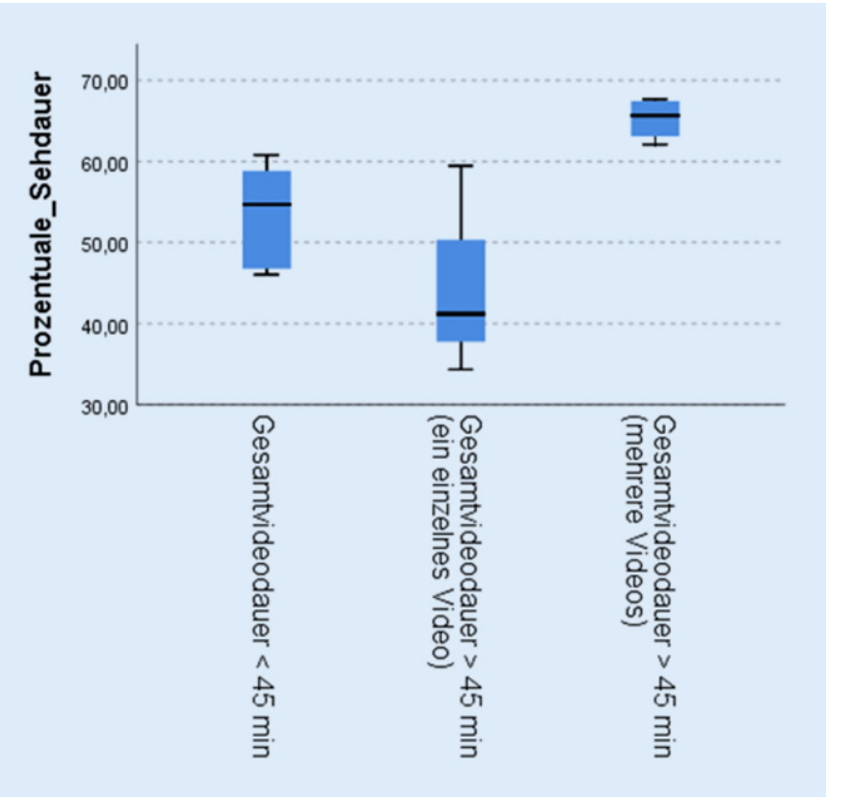

Abb. $4<$ Boxplots der prozentualen Sehdauer unterteilt nach Gesamtvideodauer und Anzahl der Videos

tervention mit insgesamt 5 Videos $1 \mathrm{~h}$ $47 \min (1: 46: 7 \mathrm{~h})$.

Die Mehrheit der Befragten bewertete (82\% stimmten [voll] zu) den zeitlichen Aufwand als angemessen (• Abb. 1).

Dennoch würden kürzere Videoabschnitte im Gegensatz zu Gesamtvideos das Lernen erleichterten (70,9\% stimmten [voll] zu; Abb. 2). Die optimale Dauer der einzelnen Videopräsentationen sollte laut der Befragten bei ca. $10-20 \mathrm{~min}$ liegen $(54,6 \%$ stimmten [voll] zu; - Abb. 3). Allerdings wünschten sich $32,7 \%$ eine längere Dauer (21-40 min) der Videopräsentationen.
Durchschnittlich betrug die Sehdauer ca. $55 \%$ der Gesamtvideodauer. Dabei zeigten sich Unterschiede, abhängig davon, ob für das Seminarthema ein einziges langes Video ( $>45 \mathrm{~min}$ ), mehrere Videos mit einer insgesamt langen Videodauer ( $>45 \mathrm{~min}$ ) oder ein oder mehrere Videos mit einer insgesamt kurzen Videodauer ( $<45 \mathrm{~min}$ ) erstellt wurden.

Die durchschnittliche prozentuale Sehdauer betrug respektive 45,0\%, $65,3 \%$ und 53,4\% (•Abb. 4). Zwischen der prozentualen Sehdauer bei einem einzelnen Video >45 min und mehreren Videos mit einer Gesamtvideodauer
$>45$ min bestand ein signifikanter Unterschied $(p=0,047)$.

Die Videopräsentationen wurden im Durchschnitt 408-mal aufgerufen, wobei das Abdomenseminar mit 618 Aufrufen am häufigsten vertreten war. Die wenigsten Aufrufe verzeichneten die Physikseminare mit 233 und 239 Aufrufen.

Die verkürzte Dauer der Videokonferenzseminare (45 statt $90 \mathrm{~min}$ ) wurde als angemessen angesehen $(86,3 \%$ stimmten [voll] $\mathrm{zu}$ ).

Allerdings zeigte eine Evaluation der tatsächlichen Onlineseminarzeiten, dass zwei Drittel der Dozenten die Seminarzeit nicht oder nicht wesentlich verkürzte. Im Minimum lag sie bei den anvisierten $45 \mathrm{~min}$, maximal betrug sie $90 \mathrm{~min}$ (durchschnittlich 77,5 min).

Mehr als die Hälfte der Teilnehmer gab aber an, dass die Videokonferenzseminare zusätzliches Wissen zu den Videopräsentationen vermittelt hätten $(53,6 \%$ stimmten [voll] zu, 33,6\% waren unentschieden, $12,7 \%$ stimmten [überhaupt] nicht $\mathrm{zu}$ ).

Der Aussage, dass das neue onlinebasierte Lehrkonzept ihr Interesse an der Radiologie geweckthätte, stimmten (voll) $72,7 \% \mathrm{zu}(24,6 \%$ waren unentschieden, 2,7\% stimmten der Aussage [überhaupt] nicht zu) und 78,2\% der Teilnehmer wurden vom Lehrkonzept zum Selbststudium animiert (18,2\% waren unentschieden, 3,6\% stimmten der Aussage [überhaupt]nicht $\mathrm{zu}$ ).

Es wurde hervorgehoben, dass das neue Konzept zeitlich und örtlich unabhängiges Lernen ermöglichte $(95,6 \%$ stimmten [voll] zu, 3,6\% waren unentschieden, 0,9\% stimmten nicht zu).

Insgesamt stimmten die meisten Studierenden der Aussage zu (82,7\%), dass sie von dem neuen Lehrkonzept profitiert hätten (• Abb. 5).

Diese Ergebnisse spiegelten sich auch in der Auswertung der Freitextbewertungen wider. Insgesamt gaben 83 Studierende ein schriftliches Feedback ab. Die Mehrheit (72,3\%) hob die gute Umsetzung der Lehre in der Radiologie hervor, bewertete das neue Lehrkonzept positiv oder lobte insbesondere die zur Verfügung gestellten Videopräsentationen. Etwas über ein Drittel (34,9\%) wünschte sich die Beibehaltung oder den verstärk- 


\section{Ich habe nicht von dem neuen Lehrkonzept profitiert.}

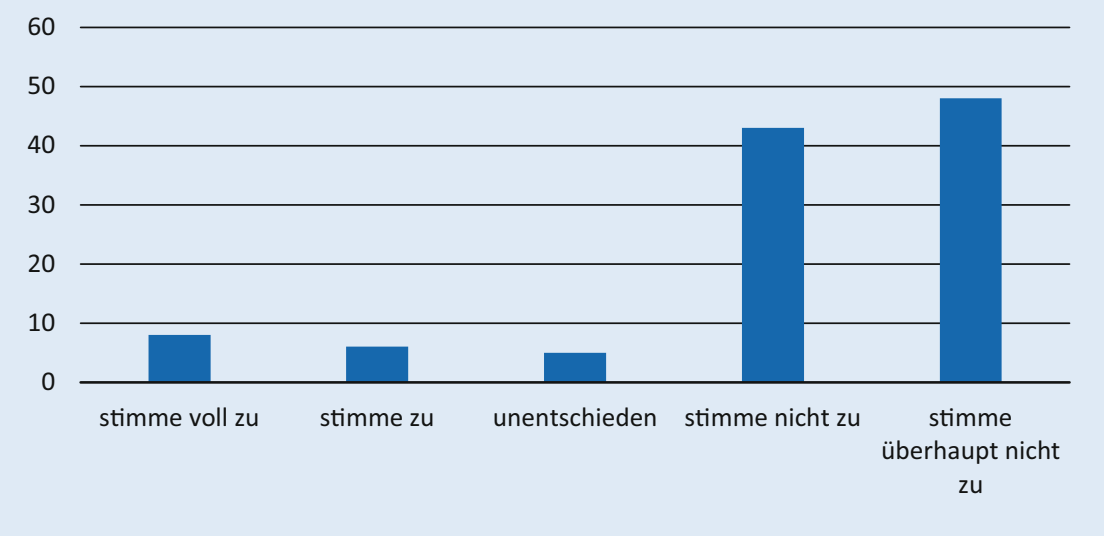

Abb. $5 \Delta$ Prozentuale Verteilung der Antworten auf die Aussage: „Ich habe nicht von dem neuen Lehrkonzept profitiert."

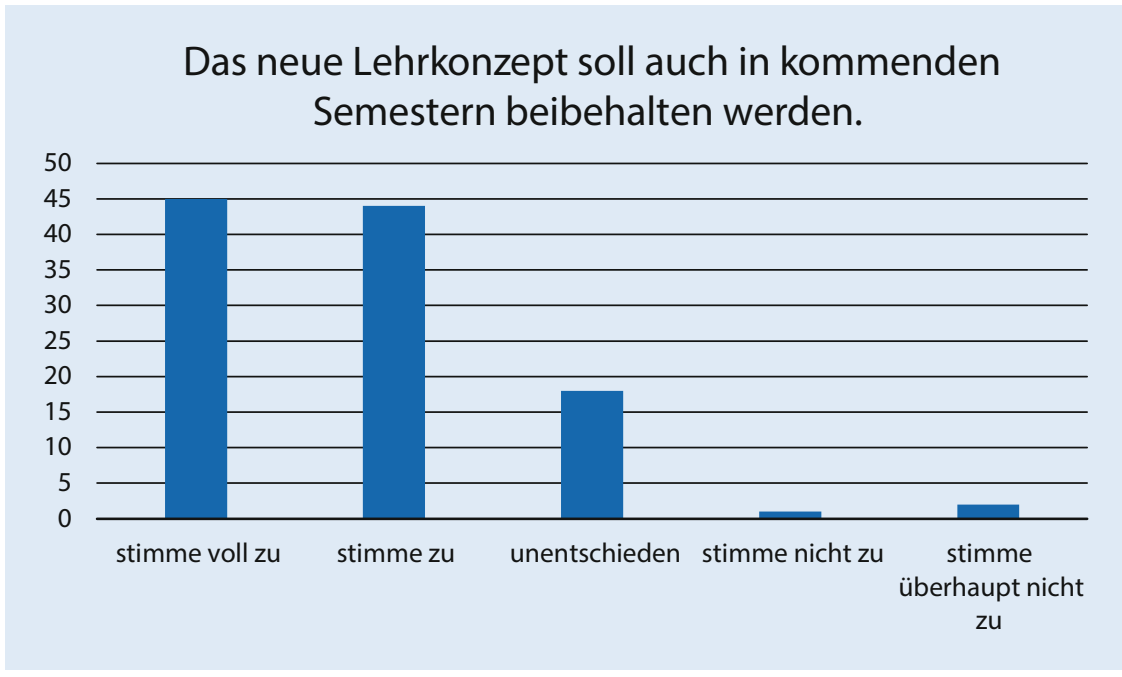

Abb. 6 A Prozentuale Verteilung der Antworten auf die Aussage: „Das neue Lehrkonzept soll auch in kommenden Semestern beibehalten werden."

ten Einsatz von Aufgabenstellungen oder Fallbeispielen zur Vorbereitung auf die Onlineseminare.

Die Teilnehmer bewerteten eine mögliche Beibehaltung des Lehrkonzeptes auch in kommenden Semestern nach Aufhebung der Kontaktbeschränkungen positiv ( $80,9 \%$ stimmten [voll] zu; - Abb. 6).

\section{Diskussion}

Durch die COVID-19-Pandemie änderten sich viele Aspekte im medizinischen Alltag. Gegenwärtig und in der nahen Zukunft spielt digitales Lernen eine zen- trale Rolle in der universitären Lehre. Unsere Ergebnisse zeigen, dass das digitale Lehrkonzept von Medizinstudierenden im Fach Radiologie gut angenommen wird.

Dies ist durch mehrere Faktoren bedingt. Wie frühere Studien bereits zeigten, beruht die Akzeptanz für neue Lehrformate auf mehreren Faktoren [7]. Neben der Benutzerfreundlichkeit und dem unkomplizierten Abrufen von Onlineinhalten, verfügt das neue Lehrkonzept über weitere wesentliche Vorteile gegenüber dem traditionellen Lehrsystem. In erster Linie handelt es sich um die zeitliche und örtliche Unabhängigkeit.
Weiterhin spielt die unbegrenzte Wiederholbarkeit der Präsentationen für das Erlernen neuen Wissens eine wichtige Rolle [1].

Die Dozenten wählten eine unterschiedliche Herangehensweise für den Aufbau der Videopräsentationen. Die Erstellung von Gesamt- oder Einzelvideos hing davon ab, ob die Dozenten ein Vorlesungsformat bevorzugten oder die Seminare von mehreren Dozenten gehalten wurden, es klare thematische Unterschiede in einem Seminar gab, wie beispielsweise beim Thema: „Angiographie und Intervention " oder Inhalte nach Normalbefund und Pathologie unterteilt wurden.

Die zeitliche Diskrepanz zwischen der durchschnittlichen Dauer der Videoseminare und der Sehdauer lässt zwei mögliche Schlüsse zu. Zum einen ist denkbar, dass kürzere Videos tatsächlich eher in Gänze angesehen wurden, während bei längeren Videos möglicherweise abgebrochen oder vorgespult wurde. Zum anderen gibt es Hinweise darauf, dass bei längeren Videos eher von der Möglichkeit des Abspielens in bis zu 2-facher Geschwindigkeit Gebrauch gemacht wurde. Dies stellt grundsätzlich kein Problem dar, allerdings sollte für die zukünftige Erstellung von Videopräsentationen bedacht werden, dass insbesondere bei der Demonstration von radiologischen Bildern hierdurch Inhalte verloren gehen könnten.

Besonders interessant ist dabei auch der ermittelte prozentuale Unterschied der durchschnittlichen Sehdauer zwischen einzelnen langen Gesamtvideos $(45,0 \%)$ und mehreren Videos mit einer insgesamt langen Videodauer (65,3\%). Geht man grundsätzlich von einer erhöhten Abspielgeschwindigkeit aus, lässt sich annehmen, dass selbst bei langer Gesamtvideodauer für ein Seminarthema die einzelnen Abschnitte eher in normaler bis leicht erhöhter Geschwindigkeit, dagegen lange Einzelvideos in mutmaßlich doppelter Geschwindigkeit und teilweise auch nicht in Gänze angesehen wurden.

Daher ist es vermutlich von Vorteil, die Videopräsentationen in kürzere $\mathrm{Ab}$ schnitte zu unterteilen. Zumal die Mehrheit der befragten Studenten der Aussage 
zustimmte, dass kürzere Videoabschnitte das Lernen erleichtern würden.

Ein weiterer wichtiger Aspekt des Lehrkonzepts ist auch, dass trotz räumlicher Entfernung Interaktivität und Dialog bei den digitalen Seminaren erhalten bleiben. Die Videokonferenzseminare bieten gute Diskussions- und Austauschmöglichkeit zwischen Dozent und Studierenden. In dem für die Onlineseminare gewählten Programm besteht die Möglichkeit zur Bildschirmfreigabe, parallelem Chat sowie zur Erstellung von Live-Befragungen, wobei die Bildschirmfreigabe und der Chat intensiv genutzt wurden.

Ein besonderer Aspekt im Fach Radiologie ist dabei auch die verbesserte Vermittlung von Kenntnissen zur Bildbefundung. Die Studierenden konnten sich im Vorfeld oder während des Seminars mit echten Fällen vertraut machen und die Erkenntnisse mit dem Dozenten per Video teilen und diskutieren. Bildinformationen und Werkzeuge zur Veranschaulichung standen dabei allen Studierenden durch die Möglichkeit zur Bildschirmfreigabe zur Verfügung, und interessante Aspekte konnten direkt für alle Seminarteilnehmer demonstriert werden.

\section{Limitationen}

Eine Limitation unserer Arbeit ist, dass von den 213 Studierenden im Kurs „Bildgebende Verfahren“ nur etwas über die Hälfte an unserer Umfrage teilgenommen haben. Bei diesen Studierenden könnte es sich um insgesamt engagiertere Studierende mit Interesse für Radiologie handeln, die ein positives Bild der digitalen Lehre zeichnen.

Nach unserem Kenntnisstand ist dies allerdings eine der ersten Arbeiten im deutschsprachigen Raum mit vergleichbaren Ergebnissen zu Studien aus Amerika und Großbritannien bezüglich der Akzeptanz und den Vorteilen der Onlinelehre, aber auch einem besonderen Augenmerk auf die videoassistierte Lehre $[2-6,11]$.

Wir führten die Befragung 5 Wochen nach Beginn des Semesters und nicht nach Abschluss des Kurses durch.
Durch mangelnde Erfahrungen im Bereich der Onlinelehre wollten wir diese Evaluation nutzen, um Konzeptänderungen noch während des laufenden Semesters vornehmen zu können. So wiesen wir nach Auswertung der Ergebnisse darauf hin, die zeitliche Verkürzung der Onlineseminare zu beachten.

Die wichtigste Erkenntnis unserer Arbeit ist aber, dass das Konzept die Mehrheit der Befragten zum Selbststudium angeregt und ihr Interesse für die Radiologie geweckt hat, sodass sich die absolute Mehrheit der Befragten die Beibehaltung des neuen Lehrkonzeptes für kommende Semester auch nach Aufhebung der Kontaktbeschränkungen im Fach Radiologie wünschte.

Explizit hervorgehoben wurde aber, dass Hospitationen und Vermittlung von Fertigkeiten wie Sonographie dennoch nicht vernachlässigt werden dürften.

Dies stellt eine große Herausforderung für die medizinische Lehre während andauernder Kontaktbeschränkungen dar.

\section{Ausblick}

Das onlinebasierte Lehrkonzept im Fach Radiologie als Notlösung in der COVID19-Pandemie genießt eine hohe Akzeptanz unter den Studierenden. $\mathrm{Zu}$ den traditionellen Lehrmethoden bietet es Vorteile wie ortsunabhängiges Lernen und bessere Möglichkeiten des Selbststudiums und sollte laut der Mehrheit der befragten Studierenden auch in kommenden Semestern nach Aufhebung der Kontaktbeschränkungen beibehalten werden.

Auch wenn es sich bei dieser Arbeit nur um die Evaluation eines Kurses handelt, zeigen die Daten, dass onlinebasierte Lehrkonzepte in der Radiologie vielfältig anwendbar sind, und es sich dabei, vergleichbar zu Studien aus Amerika und Großbritannien [2-6, 11], um ein zukunftsträchtiges Konzept handelt. Daher sollte man an der weiteren Optimierung der Onlinelehre arbeiten.

Wir werden daher die Dauer der Videopräsentationen optimieren und, sofern noch nicht geschehen, die Videos in klar unterteilte Abschnitte gliedern. Weiterhin werden wir verstärkt auf die Verkürzung der Onlineseminare achten, um die Gesamtunterrichtsdauer nicht $\mathrm{zu}$ überschreiten. Zusätzlich wünschten sich viele Studierende den noch verstärkteren Einsatz zur Bearbeitung von medizinischen Fällen und Fragestellungen zu Hause, die dann im Onlineseminar aufgelöst werden könnten. Um die Onlinelehre noch interaktiver zu gestalten, sollen in Zukunft auch Liveumfragen während der Videokonferenzen durchgeführt werden.

\section{Fazit für die Praxis}

\section{- Die onlinebasierte Lehre sollte un- serer Ansicht nach aus einer Kom- bination von Videopräsentationen zur Vorbereitung sowie interaktiven Onlineseminaren bestehen. \\ - Die Videopräsentationen sollten in kurze, klar gegliederte Abschnitte unterteilt werden und eine Möglich- keit zur Bearbeitung von Fällen oder Fragestellungen beinhalten.}

\section{Korrespondenzadresse}

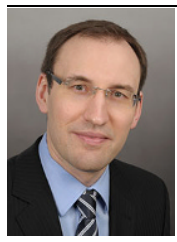

Alexey Surov

Klinik für Radiologie und Nuklearmedizin, Ottovon-Guericke Universität Magdeburg Leipziger Str 44, 39112 Magdeburg, Deutschland Alexey.Surov@med.ovgu.de

\section{Einhaltung ethischer Richtlinien}

Interessenkonflikt. A. Surov, C. March und M. Pech geben an, dass kein Interessenkonflikt besteht.

Für diesen Beitrag wurden von den Autoren keine Studien an Menschen oder Tieren durchgeführt. Für die aufgeführten Studien gelten die jeweils dort angegebenen ethischen Richtlinien.

\section{Literatur}

1. Augustin M (2014) How to learn effectively in medical school: test yourself, learn actively, and repeat in intervals. Yale J Biol Med 87:207-212

2. Darras KE, Spouge RJ, de Bruin ABH et al (2020) Undergraduate radiology education during the COVID-19 pandemic: a review of teaching and learning strategies. Can Assoc Radiol J. https://doi. org/10.1177/0846537120944821

3. DePietro DM, Santucci SE, Harrison NE et al (2020) Medical student education during the COVID19 pandemic: initial experiences implementing a virtual interventional radiology elective course. 


\section{Originalien}

Acad Radiol. https://doi.org/10.1016/j.acra.2020. 10.005

4. Durfee SM, Goldenson RP, Gill RR et al (2020) Medical student education roadblock due to COVID-19: virtual radiology core clerkship to the rescue. Acad Radiol 27:1461-1466. https://doi. org/10.1016/j.acra.2020.07.020

5. Elsayes KM, Marks RM, Kamel S et al (2020) Online liver imaging course; pivoting to transform radiology education during the SARS-CoV-2 pandemic. Acad Radiol. https://doi.org/10.1016/j. acra.2020.10.001

6. Gomez E, Azadi J, Magid D (2020) Innovation born in isolation: rapid transformation of an inperson medical student radiology elective to a remote learning experience during the COVID-19 pandemic. Acad Radiol 27:1285-1290. https://doi. org/10.1016/j.acra.2020.06.001

7. Grabowski E, Alten F, Termühlen J et al (2020) Analysis of the importance of e-learning in ophthalmology and evaluation of an e-learning app. Ophthalmologe. https://doi.org/10.1007/ s00347-020-01100-x

8. Hekmat K, Bruns CJ (2020) The impact of the COVID-19 pandemic on surgery for malignant diseases. Chirurg 91:676. https://doi.org/10.1007/ s00104-020-01238-z

9. Kriegmair MC, Kowalewski KF, Lange B et al (2020) Urology in the corona-virus pandemic - a guideline 4/20. Urologe 59:442-449. https://doi.org/10. 1007/s00120-020-01200-1

10. Matrajt L, Leung T (2020) Evaluating the effectiveness of social distancing interventions to delay or flatten the epidemic curve of coronavirus disease. Emerging Infect Dis 26:1740-1748. https://doi. org/10.3201/eid2608.201093

11. Nickinson ATO, Carey F, Tan K et al (2020) Has the COVID-19 pandemic opened our eyes to the potential of digital teaching? A survey of UK vascular surgery and interventional radiology trainees. Eur J Vasc Endovasc Surg. https://doi.org/ 10.1016/j.ejvs.2020.09.010

12. VoPham T, Weaver M, Hart J et al (2020) Effect of social distancing on COVID-19 incidence and mortality in the US. medRxiv. https://doi.org/10 $1101 / 2020.06 .10 .20127589$

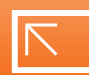

\section{Lesen Sie Ihre Zeitschrift online auf SpringerMedizin.de}

SpringerMedizin.de bietet Ihnen Zugang zu allen elektronisch verfügbaren Ausgaben und dem CME-Angebot Ihrer Zeitschrift - unabhängig davon, seit wann Sie die Zeitschrift abonniert haben.

So einfach erhalten Sie Zugang zum Online-Archiv:

- Registrieren Sie sich einmalig auf www.springermedizin.de/register

Geben Sie dabei Ihre Einheitliche Fortbildungsnummer (EFN) an.

- Ihr Benutzername entspricht Ihrer E-Mail-Adresse, Ihr Passwort können Sie frei wählen und später jederzeit unter "Mein Profil" ändern.

- Falls Sie bereits ein (Print-) Abonnement bei uns haben, geben Sie bei der Registrierung die Lieferadresse Ihrer Zeitschrift an. Damit wird Ihr Abo-Zugang auf springermedizin.de freigeschaltet.

Sind Sie bereits bei SpringerMedizin.de registriert?

Dann wird Ihr Zeitschriftenabonnement automatisch Ihrem Online-Nutzerkonto hinzugefügt. Sollten die Angaben Ihres Online-Accounts nicht eindeutig mit den Angaben Ihres Zeitschriften-Abonnements übereinstimmen, kann die Zuordnung nicht sicher erfolgen. In diesem Fall und bei allen anderen Fragen zum Online-Zugang kontaktieren Sie bitte unseren Kundenservice unter: Kundenservice@springermedizin.de

Telefonisch erreichen Sie die Hotline montags bis freitags von 9.00 bis $17.00 \mathrm{Uhr}$ kostenfrei unter 0800-77 80777 sowie gebührenpflichtig aus dem Ausland unter +4930884293600. 